\title{
SPACE ASTROMETRY AND THE HST WIDE FIELD/PLANETARY CAMERA
}

\author{
P. K. SEIDELMANN \\ U S NAVAL OBSERVATORY
}

\section{INTRODUCTION}

The launch of the Hipparcos spacecraft marked the beginning of space astrometry. Hopefully, this will be followed in the near future by the launch of the Hubble Space Telescope, which is not primarily an astrometric instrument, but has astrometric capabilities which will be described in this paper. In addition, there are plans and proposals for future astrometric spacecraft. These include the launch of a radio antenna, which combined with Earth-based antennae would provide a very, very long base line interferometer (Levy, 1986, 1988). There are proposals for launching optical interferometers, such as POINTS (Reasenberg et al 1988). There are also proposals by York and Gatewood (Gatewood et al., 1986; Gatewood 1987, 1989) for launching astrometric instruments using gratings and detectors. Thus, the future holds the prospects for a whole new capability in the field of astrometry.

\section{GROUND-BASED ASTROMETRY}

The field of ground-based astrometry can basically be divided into three categories: measurements of large angles, medium angles and small angles. Large angle measurements have been primarily restricted to brighter objects, radio sources and solar system bodies and to providing a hemisphere-wide reference system. Medium angle measurements have provided the tie between the brighter stars and fainter stars by means of astrographic catalogues. They have also provided the capability to search for unknown objects. Small angle astrometry has primarily addressed itself to parallaxes, the search for low mass companions and planetary objects and cluster dynamics.

Ground-based astrometry has been limited by the presence of an atmosphere. This controls the wavelengths that can be observed. It limits the resolution, the accuracy of the observation and generates scattered light around bright planets.

Speckle interferometry has provided the capability to observe relatively bright objects without the necessity of integrating over the atmospheric effects (McAlister 1986, 1987, $\mathrm{Lu}$, et al. 1988, McCalister et al., 1987, 1988). The introduction of the charge coupled devices as detectors to replace the eye and photographic plate has increased the dynamic range, greatly reduced the observing time and provided a detector with a built-in measuring machine. To a large extent the CCD detector was introduced, at least in my case, in anticipation of its use in space. It provides the capability of providing improved accuracies in parallaxes (Monet and Dahn 1983, Dahn et al. 1988); observing capabilities for solar system objects that are not observable by other means (Seidelmann et al. 1981, Pascu et al 1983, 1987); the potential of an improved detector on a transit circle (Gehrels et al. 1986), and the potential for establishing an inertial coordinate system (Mao, et al, 1989). 
An advantage of the $\mathrm{CCD}$ as a detector is demonstrated in the satellite observing program. With photographic plates the faint librational satellites would require long exposures and the images would then be lost in the scattered light of the planet. With a CCD the exposure lengths are reduced and, since the exposures are recorded digitally, the planetary scattered light can be subtracted based on a model. Thus, these satellites can be observed with a CCD, but not with photographic plates. Similarly, Nereid could be observed with a CCD from Flagstaff with six minute exposures that would have required with a photographic plate over an hour, a length of time that could not have been achieved with Neptune so far to the south.

\section{SPACE ASTROMETRY}

The primary advantage of doing astrometry from space is the absence of an atmosphere. Thus, the limitations imposed by the atmosphere on wavelength, resolution, scattered light, and accuracy are relieved. However, new limitations are imposed. These include the knowledge of the spacecraft orbit, the stability of the spacecraft, limitations on data rate, calibration requirements and the inability to tinker with the instrument. The accuracy and resolution limitations from space will not be imposed by the environment, but rather determined by the cleverness of the designer and the capabilities of the available hardware.

A number of papers at this meeting discuss the planned capabilities of the Hipparcos spacecraft to observe parallaxes, minor planets and triple stars. The Hipparcos spacecraft is an example of a large angle measuring device, although it is done in a different manner than from the ground (Kovalevsky 1986). The Hubble Space Telescope (HST), on the other hand, more closely resembles a ground-based instrument in space. General information about the HST has been published in a number of places in the literature (e.g. The Space Telescope Observatory, 1982). For astrometric purposes, HST includes the fine guidance sensors (FGS) that will be used to guide the telescope and can be used for astrometric positional observations. Their capability is described in another paper.

The HST has two imaging instruments. One is the Faint Object Camera which has extremely limited astrometric capabilities due to its very small field of view (maximum 45"). The other instrument, the Wide Field/Planetary Camera (WF/PC), has a large field of view compared to the faint object camera but a very small field of view compared to ground-based telescopes. The planetary camera provides a field of view of 68 arcseconds on a side with 4 CCD detectors. The Wide Field camera has a field of 160 arcseconds on a side. For astrometric purposes it is necessary to trace the path of light which enters the WF/PC by means of a pick-off mirror in the center of the field of view of the HST. The light enters through a window and passes through a filter, which is one of 48 possible selections. The light then encounters the four-faceted reflective pyramid that divides the light into four separate beams, which are directed through reimaging optics, through a field flattener, and onto a CCD. The choice between the Planetary and Wide Field modes is made by the selection of the position of the pyramid.

While the pyramid is essentially a reflecting optical component, it contains a spot with a nonreflecting coating approximately 1.23 arcsecond in diameter which is located in the middle of the field of view of one chip (PC8) of the planetary camera. It is toward the central corner of the field of view of CCD WF4. This nonreflecting Baum spot attenuates the light of an image falling on that spot. The pyramid also has very small spots along the ridges of the pyramid that are cleared of reflective coating. The pyramid can be back lit so that the light coming through one of these spots on a ridge of the pyramid appears on both of the adjoining CCD chips. This provides a means of tying the four images together in a mosaic. 


\section{CALIBRATION}

The spots, called the Kelsall spots, provide an independent means of calibration. An exposure of the Kelsall spots can: 1) confirm that the pyramid is completely' rotated into the correct position; 2) by measuring the positions of the Kelsall spots, detect whether an individual chip or reimager has moved, tilted or shifted in focus. Thus, the Kelsall spots provide an internal monitor on the WF/PC. Additional calibrations will be made by' using either Omega Centauri, or NGC 6752.

The WF/PC is known to have pin cushion distortion from the reimaging systems. This effect can reach a magnitude of $11 / 2$ pixels or 22 microns near the edge of the chip. The WF/PC also has the potential for distortions due to what is called the "potato chip effect." Since the CCD chips are thin devices, the chip may not be flat. The field flattener lens can displace the beam by 0.1 radian near the edge. The pupil is approximately 75 millimeters in front of the focal plane, as are the WF and PC field flatteners. The angle to the corner of the chip is then 0.11 radians and to the center of the edge of the chip 0.08 radians. The astrometric effects in the corner are 2.7 microns (18 milliarcseconds (mas) for WF and 8 mas for PC) and at the center of the edge 2.0 microns (13 mas for WF and 6 mas for PC).

Since the thinned chips are supported at the edges, the ripples go to zero where the effects of the field flattener are a maximum. The result of the combination of the field flattener and the ripple could be anticipated to be 1.5 micron (10 mas for the WF and 4 mas for the PC).

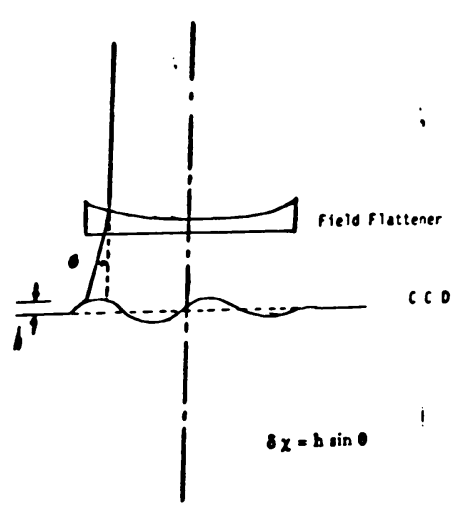

POTATO CHIP EFFECT

Figure 1
It is possible that the chip can change its shape relative to a flat surface as a function of temperature. Since there is a field flattener in front of the chip, this lack of flatness can cause a displacement in position of the image (Fig 1). A typical half amplitude of the ripple would be 25 microns and half the scale length about 100 pixels.

There is also a potential distortion of position due to the fact that there are defects in the CCD's. If an image falls on a bad pixel or a bad column, the image will be affected and the position accordingly affected. Therefore, small shifts in the pointing can be used to detect and average out shifts due to defects. These shifts also provide multiple exposures that improve the resulting accuracy as a function of the square root of the number thereof. Shifted positions also provide a means of isolating the cosmic ray events that will be present.

In addition to the changes in the shapes or flatness of the chips due to the changes in temperature, it is also possible that there will be shifts in the positions of the chips as a function of temperature. While the optical bench is made out of graphite epoxy for stability, there is a possibility of the motion of the chips due to the temperature of the cameras.

To investigate the distortions of the WF/PC, a series of observations were made during thermal vacuum test No. 6 using slant bars. Measurements of the slant bars provided a means of evaluating the third order model required for pin cushion distortion for real data. The measured displacements as determined from the differences between the 
measured positions of the slant bars and the positions of uniformly spaced, constant slope, slant bars is shown in Fig. 2. When the measurements are corrected by the third order model, the remaining residuls due to the potato chip effect and chip defects are shown in Fig. 3. Thus, it is expected that accurate positional results will require correcting the measured positions with a third order model for pin cushion distortion and a mapping of the remaining effects.

Figure 2 Fig. 10
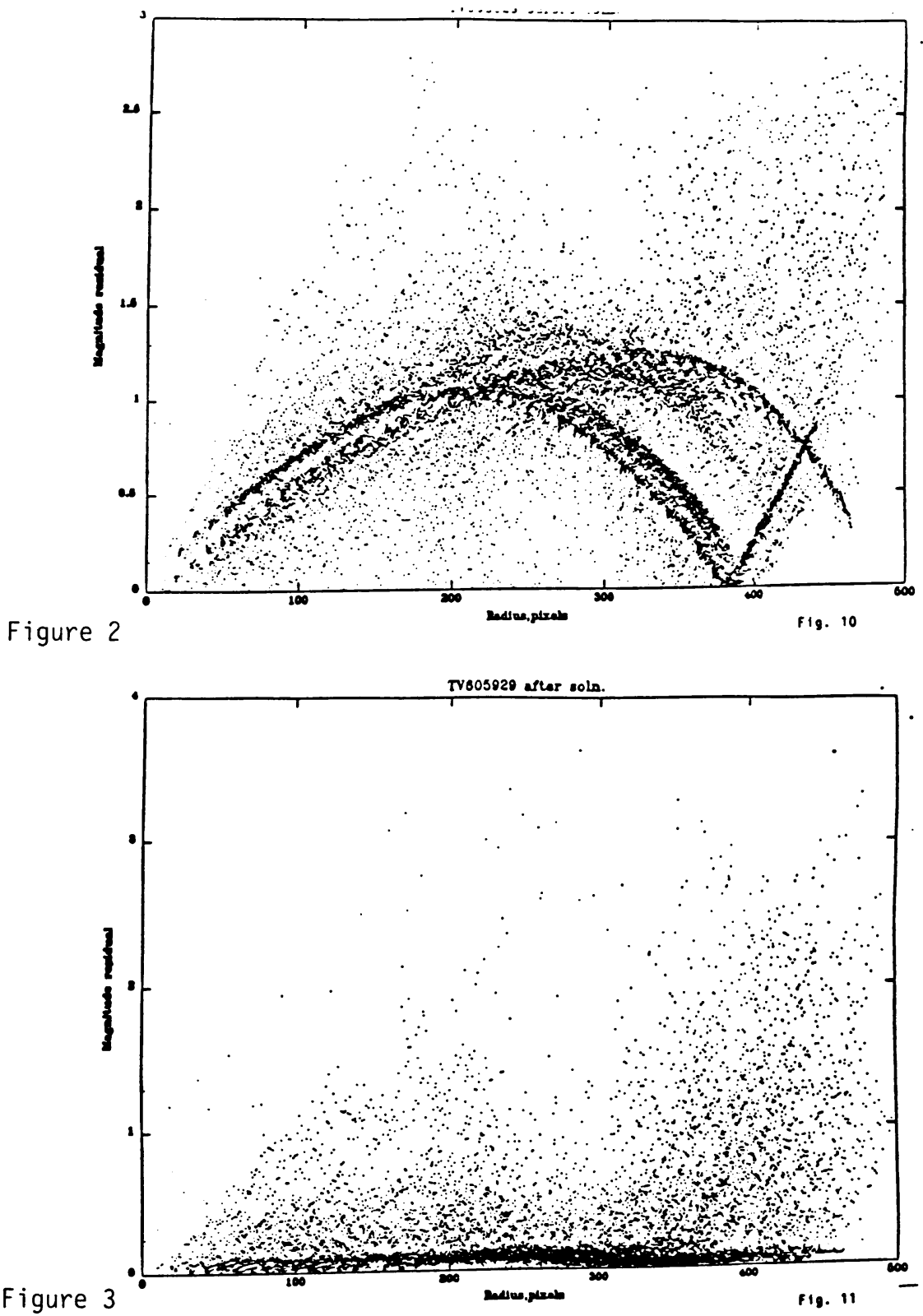

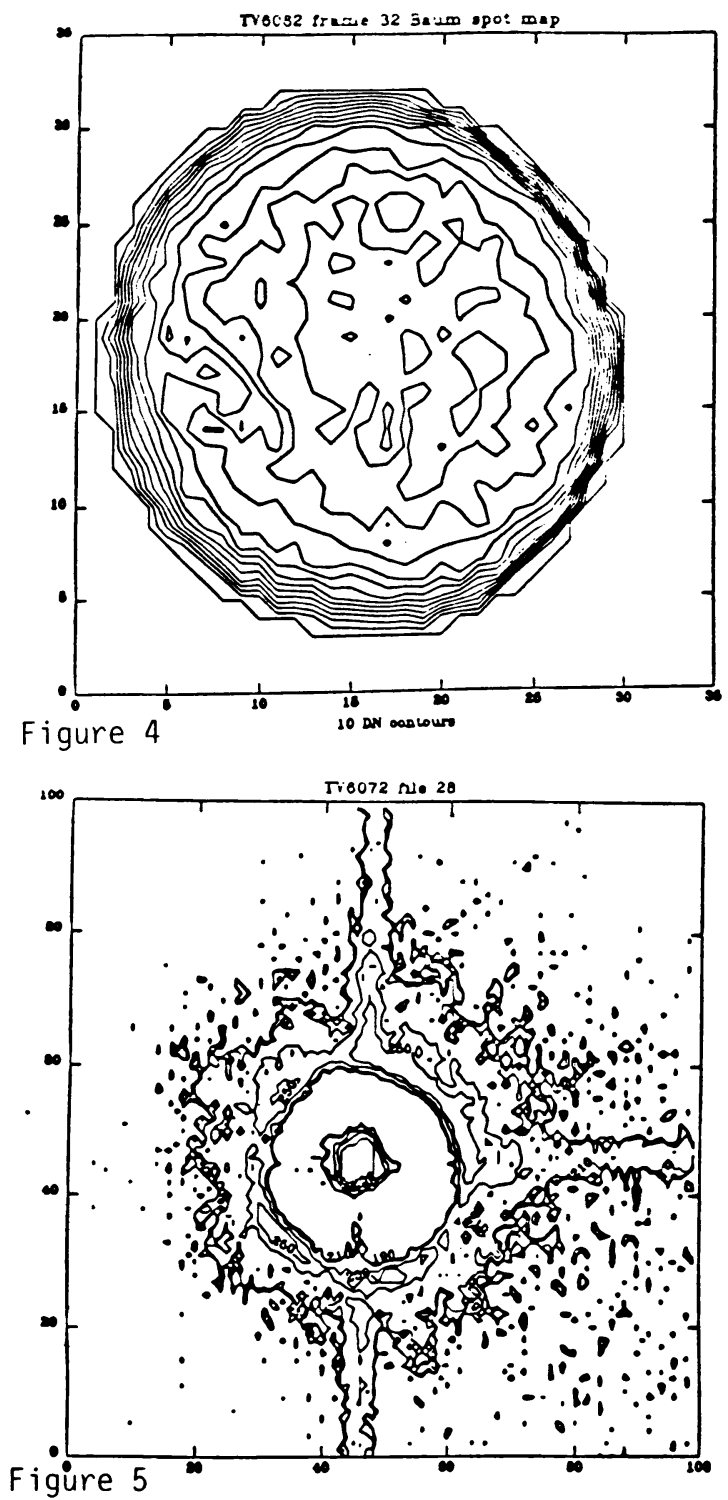

In flight, it is planned that images will be taken of a field of Omega Centauri or NGC 6752 which has a sufficient number of stars to represent the third order distortion. Shifted positions of the Omega Centauri field are designed for investigations of the potato chip effect, and changes thereof. In order to evaluate the potato chip effect observations must be made with a large number of stars covering all parts of each of the chips.

The Baum spot has a reduced reflectance that is a function of wavelength. A contour plot of the floor of the Baum spot is shown in Fig. 4. The axes are in pixels from (407, 402). The maximum $D N$ is 250. A bright spot at $(7,17)$ is $40 \mathrm{DN}$ above the floor. The reflectance of the spot was measured by placing a pinhole target spot on and off of the Baum spot in thermal vacuum tests. Preliminary results indicate that with filter F555W, the Baum spot reduces the reflectance by $6.156+0.064$ magnitudes. Figure 5 shows the contour plot of the pinhole target in the center of the Baum spot with the diffraction spikes from the reimaging system. The diffracted light outside the spot reaches approximately the same DN level as the target pinhole under the spot.

Calibration of the WF/PC for astrometric purposes requires that we know the following information:

1. Distortions due to each reimaging system.

2. Distortions of each chip.

3. Thermal stability of each chip.

4. Flat field for each chip for each filter.

5. Angle between surface of chip and optical axis.

6. Point spread function for different places on chip. 
7. "Plate constants" for each chip.

8. Location of the different chips with respect to each other.

9. Stability of chip locations.

10. Location of WF/PC optical field with respect to FGS and other instruments.

11. Stability of WF/PC with respect to other instruments.

12. Attenuation characteristics of Baum spot.

While some of these measurements can be made from thermal vacuum tests made prior to launch, the measurements must also be made in space to determine the real conditions. All stability characteristics require continued measurements to leam of changes.

\section{REDUCTION METHODS}

CCD's have been used for astrometric observations from the ground. Experience has accumulated concerning the methods of centroiding and the accuracy which can be achieved in measuring the images. In general, ground-based observations can be measured with a one dimensional fit to a Gaussian to determine centroids. Two dimensional fits do not significantly improve the resulting accuracies in most cases. As long as the signal-to-noise ratio exceeds approximately 5 , an accuracy in the neighborhood of 1/40th of a pixel can be achieved. It is desirable to increase the exposure such that the DN number of the brightest image to be measured is just below the beginning of saturation.

On the HST the Wide Field Camera is undersampled with extremely small point spread functions. The point spread function will vary somewhat over the individual chip. Experience with simulated data indicates a slight preference for the use of a Cauchy

function as opposed to a Gaussian function and indicates that the one dimensional fits are approximately as satisfactory as the two dimensional fits (Santoro et al. 1989). The errors due to the distortions, however, make the differences in the centroiding methods insignificant.

Experiences with simulations indicate that the identification and recognition of the star fields is extremely difficult. Due to the small size of the images, there is not a good visual identification of the relative magnitude of the stars. Also, cosmic ray events contribute many "volunteer stars" which are not easily identified.

Based on calibration observations from the ground and from space, it is expected that there will be a third order distortion model plus an individual chip map model for correcting the distortions. There will be solutions based on the calibration field and the Kelsall spots for combining the four chips into a single field. The accuracy and stability of that combining procedure is not known and will, to some extent, depend on the stability of the chips within the WF/PC.

The present plans for using the WF/PC for astrometry include searches for unknown satellites, observations of extremely faint satellites that cannot be observed from the ground; observations of Pluto and its satellite; observations of the rings of Jupiter, Uranus and Neptune; searches for low mass companions or planetary objects around selected stars; observations of minor planets; and observations of radio sources or quasars with respect to reference stars. There are plans for direct observations of low mass companions, but the characteristics of the HST mirrors and the instruments make the success of such observations unlikely. 


\section{CONCLUSIONS}

We are on the threshold of astrometry from space. This offers the potential for achieving observations with significantly improved accuracy and resolution. Exciting science can be expected from the improved accuracies and resolutions to be achieved from space. There will be great competition, first for funding for astrometric instruments in space; and second, for observing time for astrometric observations on more general spacecraft, such as the HST. A strong case is going to have to be made for observing from space. Hopefully, significant improvements can be achieved, both from the ground and from space with the new techniques currently available. It will be necessary to perform astrometry where it can be done most efficiently, economically and effectively.

\section{ACKNOWLEDGEMENTS}

It is my pleasure to thank Earnest Santoro, Richard Schmidt, Stephen Panossian and David Monet, who contributed material used in this paper.

\section{REFERENCES}

Bandermann, L., Bareket, N., and Metheny, W., (1982) "Comparative Feasibility Study of Two Concepts for a Space-Based Astrometric Satellite." NASA - CR-166403.

Bernstein, H. H., Hering, R., and Walter, H. G. (1988) "Astrometric Parameters of Visual Double Stars Derived from Simulated Hipparcos Measurements." Astrophys. \& Space Sci. 142, 161.

Dahn, C.C., Harrington, R. S. Kallarakal, V. V., Guetter, H. H., Luginbuhl, C. B., Riepe, B. Y., Walker, R. L., Pier, J.R., Vrba, F. J., Monet, D. G and Ables, H. D. (1988) "U. S. N. O. Parallaxes of Faint Stars." Astron. J., 95, 237.

Gatewood, G., (1987) "The Multichannel Astrometric Photometer and Atmospheric Limitations in the Measurements of Relative Positions." Astron. J. 94, 213.

Gatewood, G., (1989) "MAP Determinations of the Parallaxes of Stars in the Regions of HD 2665, BD +68 DEG 946, and Lambda Ophiuchi." Astron. J., 97, 1189.

Gatewood, G., Stein, J., Kiewiet de Jonge, J., Faste, D., and Breakiron, L (1986)

"A New Astrometric System." Astrometric Techniques: IAU Symposium 109, Florida, 341.

Gehrels, T., Marsden, B. G., McMillan, R.S., and Scott, J. V. (1986) "Astrometry

With A Scanning CCD." Astron. J. 91, 1242.

(1988) "Hipparcos' Final Test." Sky \& Telescope, Vol. 75, No. 4, Apr. p. 358.

(1989) "Hubble Space Telescope Wide Field and Planetary Camera Instrument Handbook" Version 2. Space Telescope Science Institute.

Kovalevsky, J., (1986) "Hipparcos Satellite and the Organization of the Project." Astrometric Techniques: IAU Symposium 109, Florida, 581.

Levy, E. H., Gatewoood, G. D., Stein, J. W., and McMillan, R. S. (1986) "Astrometric Telescope of 10 Microarcsecond Accuracy on the Space Station." Advanced Technology Optical Telescopes (Proc. SPIE, Int Soc, Opt Eng 628) III, 181.

Levy, G. S., (1986) "First Successful Very Long Baseline Interferometry Observations Using an Orbiting Telescope." Preprint, Nobeyama Radio Observatory Report No. 109.

Levy, G.S., (1986) "Status of the Very Long Baseline Interferometry VLBI/Demonstration Using the Tracking and Data Relay Satellite System." Preprint, Nobeyama Radio Observatory Report No. 116.

Levy, G. S., (1988) "VLBI Using a Telescope in Earth Orbit - The Observations."

JPL Astrophysics Preprint. 
Lu, P. K., Demarque, P., Van Altena, W., McAlister, H. and Hartkopf, W., (1987) "ICCD Speckle Observations of Binary Stars. III. A Survey for Duplicity among High Velocity Stars." Astron. J., 94, 1318.

Mao Wei, Guo Xinjian, Xu Shui, Wu Guangjie, and Lu Ruwei, (1989) "Construction of an Inertial Coordinate System Using a CCD," Astron. Astrophysics 215, 190.

McAlister, H. A., (1986) "Speckle Interferometry in Astrometry." Astrometric Techniques: IAU Symposium 109, Florida, 293.

McAlister, H. A., (1987) "The Future of High Angular Resolution Astronomy: Seeing the Unseen." Vistas in Astronomy, 30, 27.

McAlister, H. A., Hartkopf, W. I. and Gutter, D. J. (1987) "ICCD Speckle Observations of Binary Stars. II: Measurements During 1982 - 1985 from the Kitt Peak 4m Telescope." Astron. J., 93, 688.

McAlister, H. A., Hartkopf, W. J., Bagnuolo, W. G., Sowell, J. R., Franz, O. G. and Evans, D. S. (1988) "Binary Star Orbits From Speckle Interferometry - I. The Hyades Binary Finsen 342 (70 Tauri)." Astron. J. 96, 1431.

Monet, D. G. and Dahn, C.C. (1983) "CCD Astrometry. I. Preliminary Results from the KPNO 4-m/CCD Parallax Program" Astron. J., 818, 1489.

Pascu, D., Seidelmann, P. K., Baum, W. A., and Schmidt, R.E. (1983) "Observations of Faint Planetary Satellites with Charge-Coupled Device."In The Motion of Planets and Natural and Artificial Satellites, edited by S. Ferraz-Mello and P. E. Nacozy, (Universidade de Sao Paulo, Sao Paulo, Brazil), 253.

Pascu, D., Seidelmann, P. K., Schmidt, R.E., Santoro, E. J., and Hershey, J. L. (1987), "Astrometric CCD Observations of Miranda: 1981 - 1985." Astron. J., 93, $963-968$.

Reasenberg, R. D., Babcock, R. W., Chandler, J. F., Gorenstein, M. V., Huchra, J. P., Pearlman, M. R., Shapiro, I. I., Taylor, R. S., Bender, P., Buffington, A., Carney, B., Hughes, J. A., Johnston, K. J., Jones, B. F., and Matson, L. E., (1988) "Microarcsecond Optical Astrometry: An Instrument and its Astrophysical Applications," Astron. J. 96, 1731.

Santoro, E. J., Schmidt, R. E., Seidelmann, P. K., and Kristian, J., (1989) Centroid Analysis of Space Telescope Widefield Camera Point Spread Function Images" In Errors, Bias, and Uncertainties in Astronomy, Strasbourg, France.

Seidelmann, P. K. , Harrington, R. S., Pascu, D., Baum, W. A., Currie, D. G., Westphal, J. A., and Danielson, G. E. (1981) Saturn Satellite Observations and Orbits from the 1980 Ring Plane Crossing. Icarus, 47, 282.

"The Space Telescope Observatory," (1982) edited by N. B. Hall, Space Telescope Science Institute. 